\title{
Accurate classification of 17 AGNs detected with Swift/BAT ${ }^{\star}$
}

\author{
P. Parisi ${ }^{1,2}$, N. Masetti ${ }^{1}$, E. Jiménez-Bailón ${ }^{3}$, V. Chavushyan ${ }^{4}$, A. Malizia ${ }^{1}$, R. Landi ${ }^{1}$, M. Molina ${ }^{5}$, M. Fiocchi ${ }^{6}$, \\ E. Palazzi ${ }^{1}$, L. Bassani ${ }^{1}$, A. Bazzano ${ }^{6}$, A. J. Bird ${ }^{7}$, A. J. Dean ${ }^{7}$, G. Galaz ${ }^{8}$, E. Mason ${ }^{9}$, D. Minniti ${ }^{8}, 10$, L. Morelli ${ }^{11}$, \\ J. B. Stephen ${ }^{1}$, and P. Ubertini ${ }^{6}$
}

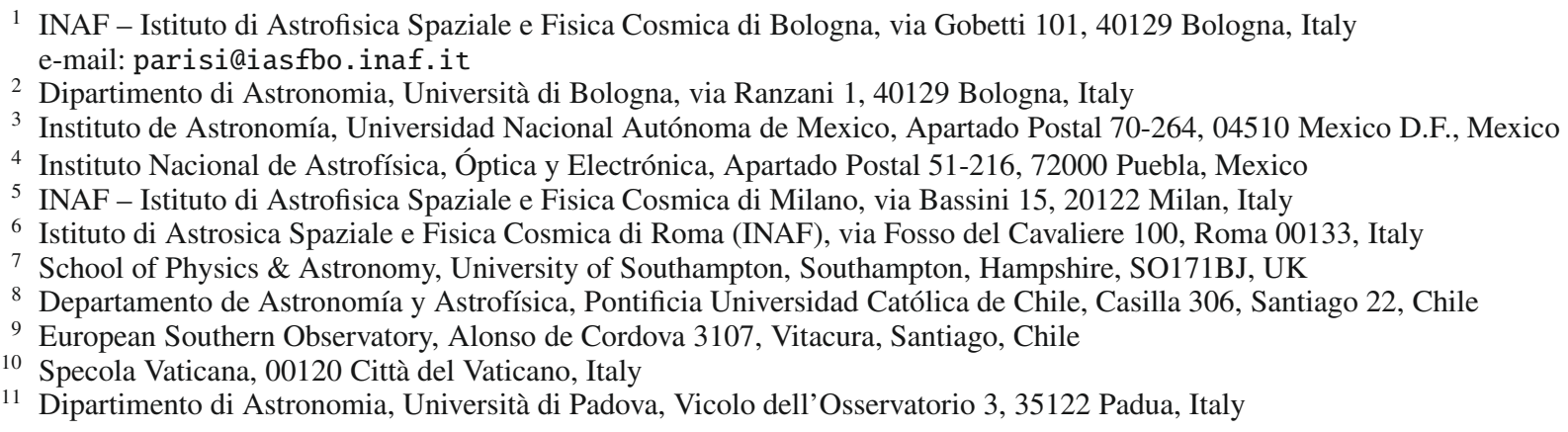

Received 20 July 2009 / Accepted 12 September 2009

\section{ABSTRACT}

\begin{abstract}
Through an optical campaign performed at 5 telescopes located in the northern and the southern hemispheres, plus archival data from two on line sky surveys, we have obtained optical spectroscopy for 17 counterparts of suspected or poorly studied hard X-ray emitting active galactic nuclei (AGNs) detected with Swift/BAT in order to determine or better classify their nature. We find that 7 sources of our sample are type 1 AGNs, 9 are type 2 AGNs, and 1 object is an X-ray bright optically normal galaxy; the redshifts of these objects lie in a range between 0.012 and 0.286 . For all these sources, X-ray data analysis was also performed to estimate their absorption column and to search for possible Compton thick candidates. Among our type 2 objects, we did not find any clear Compton thick AGN, but at least 6 out of 9 of them are highly absorbed $\left(N_{\mathrm{H}}>10^{23} \mathrm{~cm}^{-2}\right)$, while one does not require intrinsic absorption; i.e., it appears to be a naked Seyfert 2 galaxy.
\end{abstract}

Key words. galaxies: Seyfert - techniques: spectroscopic

\section{Introduction}

The Swift mission was designed to study cosmic gamma-ray bursts (GRBs) in a multiwavelength context (Gehrels et al. 2004). The aims of this mission are to determine of the origin of these phenomena and to search for new types of GRBs, including their interaction with the surrounding medium and their use as probes to study the universe at $z>5$.

Swift, with its unique repointing capabilities, is able to study and monitor other types of X-ray emitting objects. Through its payload, consisting of three instruments, i.e. the burst alert telescope (BAT; Barthelmy 2004), the X-Ray telescope (XRT; Burrows et al. 2004) and the ultraviolet/optical telescope (UVOT; Roming et al. 2004), Swift can detect and follow up $\mathrm{X}$-ray emitting objects up over a wide range of wavelengths.

In particular, BAT is a coded mask instrument operating in the energy range 14-195 keV, with a field of view of $1.4 \mathrm{sr}$, and is able to provide a source position determination with an

^ Based on observations obtained from the following observatories: Telescopio Nazionale Galileo at the Observatorio del Roque de los Muchachos of the Instituto de Astrofísica de Canarias (Canary Islands, Spain); Astronomical Observatory of Bologna in Loiano (Italy); Astronomical Observatory of Asiago (Italy); Cerro Tololo Interamerican Observatory (Chile); Observatorio Astronómico Nacional (San Pedro Mártir, Mexico). uncertainty of $1^{\prime}-4^{\prime}$ (Gehrels et al. 2004) depending on source intensity. Its sensitivity is estimated at $\sim 1 \mathrm{mCrab}$ at high Galactic latitudes and $\sim 3 \mathrm{mCrab}$ for strong sources on the Galactic plane.

As said before, this instrument is not only able to detect new GRBs, but also to perform a highly sensitive hard X-ray survey of the sky (e.g. Cusumano et al. 2009). Indeed, the BAT surveys of Tueller et al. $(2008,2009)$ allow, in particular, study of the extragalactic X-ray sky, and the observation of absorbed AGNs over a range of energies not affected by absorption due to intervening material.

Observations performed below $10 \mathrm{keV}$ with other satellites, such as ASCA, BeppoSAX (Matt et al. 2000; Ueda et al. 1999), Chandra, XMM, and Suzaku (Ueda et al. 2007; Guainazzi et al. 2005), have revealed a population of absorbed AGNs with a hydrogen column density along the line-of-sight higher than $10^{23} \mathrm{~cm}^{-2}$, which obscures the nuclei at optical and soft $(0.2-10 \mathrm{keV}) \mathrm{X}$-ray bands. Quantifying the number of these particular objects, especially at low redshifts, is very important if one wants to understand the accretion mechanisms at work in AGNs and how the absorbed AGNs contribute to the cosmic X-ray background (Comastri et al. 2004).

Now a number of surveys at energies higher than $10 \mathrm{keV}$ are available to study this class of objects. The surveys performed by IBIS (Ubertini et al. 2003) on board INTEGRAL (Winkler et al. 2003), together with those of BAT, provide the best sample of 
Table 1. Log of the spectroscopic observations presented in this paper (see text for details).

\begin{tabular}{|c|c|c|c|c|c|c|c|}
\hline $\begin{array}{c}(1) \\
\text { Object }\end{array}$ & $\begin{array}{c}(2) \\
\text { RA } \\
(\mathrm{J} 2000) \\
\end{array}$ & $\begin{array}{c}(3) \\
\text { Dec } \\
(\mathrm{J} 2000) \\
\end{array}$ & $\begin{array}{c}(4) \\
\text { Telescope+instrument }\end{array}$ & $\begin{array}{c}(5) \\
\lambda \text { range } \\
(\AA)\end{array}$ & $\begin{array}{c}\text { (6) } \\
\text { Disp. } \\
(\AA / \text { pix })\end{array}$ & $\begin{array}{c}\text { (7) } \\
\text { UT date \& time } \\
\text { at mid-exposure }\end{array}$ & $\begin{array}{c}(8) \\
\text { Exposure } \\
\text { time (s) }\end{array}$ \\
\hline Swift J0059.4+3150 & 005953.28 & +314937.3 & SPM 2.1m+B\&C Spc. & $3450-7650$ & 4.0 & 30 Jun. $2008,10: 50$ & 1800 \\
\hline Swift J0134.1-3625 & 013357.75 & -362935.8 & CTIO 1.5m+RC Spc. & $3300-10500$ & 5.7 & 02 Jul. 2008, 09:02 & 1200 \\
\hline Swift J0342.0-2115 & 034203.71 & -211439.6 & $\mathrm{AAT}+6 \mathrm{dF}$ & $3900-7600$ & 1.6 & 30 Nov. $2003,13: 34$ & $1200+600$ \\
\hline Swift J0350.1-5019 & 035023.78 & -501835.5 & CTIO 1.5m+RC Spc. & $3300-.10500$ & 5.7 & 04 Mar. 2008, 01:23 & $2 \times 1800$ \\
\hline Swift J0505.7-2348 & 050545.75 & -235114.0 & SPM $2.1 \mathrm{~m}+\mathrm{B} \& \mathrm{C}$ Spc. & $3450-7650$ & 4.0 & 03 Feb. 2008, 04:04 & $2 \times 1800$ \\
\hline Swift J0501.9-3239* & 051935.81 & -323928.0 & $\mathrm{AAT}+6 \mathrm{dF}$ & $3900-7600$ & 1.6 & 30 Jan. 2003, 10:36 & $1200+600$ \\
\hline Swift J0640.1-4328 & 064037.99 & -432121.1 & CTIO $1.5 \mathrm{~m}+\mathrm{RC}$ Spc. & $3300-10500$ & 5.7 & 05 Mar. 2008, 1:26 & $2 \times 1800$ \\
\hline Swift J0727.5-2406 & 072721.05 & -240632.3 & TNG+DOLoRes & $3800-8000$ & 2.5 & 07 Feb. 2008, 23:35 & $2 \times 1200$ \\
\hline Swift J0739.6-3144 & 073944.69 & -314302.5 & SPM 2.1m+B\&C Spc. & $3450-7650$ & 4.0 & 03 Feb. 2008, 07:15 & $2 \times 1800$ \\
\hline Swift J0743.0-2543 & 074314.72 & -254550.1 & CTIO $1.5 \mathrm{~m}+\mathrm{RC}$ Spc. & $3300-10500$ & 5.7 & 04 Mar. 2008, 03:56 & $2 \times 1200$ \\
\hline Swift J0811.5+0937 & 081130.83 & +093350.9 & TNG+DOLoRes & $3800-8000$ & 2.5 & 08 Feb. 2008, 00:51 & $2 \times 1800$ \\
\hline Swift J0902.0+6007 & 090158.37 & +600906.2 & Copernicus+AFOSC & $4000-8000$ & 4.2 & 22 Feb. 2008, 21:07 & $1200+600$ \\
\hline Swift J0904.3+5538 & 090436.97 & +553602.6 & SDSS+CCD Spc. & 3800-9200 & 1.0 & 29 Dec. $2000,10: 48$ & 9000 \\
\hline Swift J0911.2+4533 & 091130.00 & +452806.0 & SDSS+CCD Spc. & 3800-9200 & 1.0 & 07 Feb. 2002, 08:24 & 4803 \\
\hline Swift J0917.2-6221 & 091609.37 & -621929.6 & CTIO $1.5 \mathrm{~m}+\mathrm{RC}$ Spc. & $3300-10500$ & 5.7 & 04 Mar. 2008, 06:02 & 600 \\
\hline Swift J0923.7+2255 & 092343.01 & +22 5432.4 & SPM $2.1 \mathrm{~m}+\mathrm{B} \& \mathrm{C}$ Spc. & $3450-7650$ & 4.0 & 02 Feb. 2008, 11:23 & 1800 \\
\hline Swift J1049.4+2258 & 104930.89 & +225752.4 & Cassini+BFOSC & $3500-8000$ & 4.0 & 08 May $2008,21: 17$ & $2 \times 1200$ \\
\hline
\end{tabular}

Source coordinates are extracted from the 2 MASS catalog and have an accuracy better than $0.1 .{ }^{*}$ The name of this source reported in Tueller et al. (2009) does not correspond to the actual coordinates.

objects selected in the soft gamma-ray band to date (Bird et al. 2007; Krivonos et al. 2007). IBIS and BAT work in similar spectral bands, but concentrate on different parts of the sky: IBIS maps mainly the Galactic plane, while BAT focuses on observations at high Galactic latitudes.

The nature of AGNs detected in these surveys is however often not confirmed and sometimes just assumed on the basis of their X-ray spectrum; therefore an optical follow-up of these sources is required. The optical spectra are not only crucial for an accurate classification, but can provide fundamental parameters which together with softness flux ratio (as in Malizia et al. 2007), can give us information about their possible Compton thick nature (an AGN is defined as "Compton thick" when the column density along its line of sight is equal to or greater than the inverse of the Thomson cross-section, i.e. $N_{\mathrm{H}} \geq 1.5 \times 10^{24} \mathrm{~cm}^{-2}$ ).

In this work we have selected from the Swift/BAT AGN surveys of Tueller et al. (2009), Ajello et al. (2008) and Winter et al. (2008) those objects (17 in total) either without optical identification, or not well studied or without published optical spectra. Following the method applied by Masetti et al. (2004, 2006a,b, $2008,2009)$ for the optical spectroscopic follow-up of unidentified INTEGRAL sources, we determine the nature of the 17 selected objects which are listed in Table 1.

We show here the spectroscopic results obtained on this sample at various telescopes, thanks to an observational multisite campaign carried out in Europe, Central and South America plus the use of archival spectra available online. In Sect. 2 we give a description of the observations and the employed telescopes, with information on the data reduction method. Section 3 reports the X-ray data analysis for all of our sources. Section 4 reports and discusses the results for each individual source together with general characteristics (central black hole masses and Compton thickness). In Sect. 5 comments and conclusions are given.

\section{Optical spectroscopy}

The following telescopes were used for the optical spectroscopic study presented here:

- the $1.5 \mathrm{~m}$ telescope at the Cerro Tololo Interamerican Observatory (CTIO), Chile;
- the $1.52 \mathrm{~m}$ "Cassini" telescope of the Astronomical Observatory of Bologna, in Loiano, Italy;

- the 1.8m "Copernicus" telescope at the Astrophysical Observatory of Asiago, in Asiago, Italy;

- the $2.1 \mathrm{~m}$ telescope of the Observatorio Astrónomico Nacional in San Pedro Martir, Mexico;

- the 3.58m telescope “Telescopio Nazionale Galileo" (TNG) at the Observatorio of the la Roque de Los Muchachos (Canary Islands, Spain).

The data reduction was performed with the standard procedure (optimal extraction; Horne 1986) using IRAF $^{1}$. Calibration frames (flat fields and bias) were taken on the day preceeding or following the observing night. The wavelength calibration was obtained using lamp spectra acquired soon after each on-target spectroscopic acquisition; the uncertainty on the calibration was $\sim 0.5 \AA$ for all cases; this was checked using the positions of background night sky lines. Flux calibration was performed using catalogued spectrophotometric standards. Objects with more than one observation had their spectra stacked together to increase the signal-to-noise ratio.

Further spectra were retrieved from two different astronomical archives: the Sloan Digitized Sky Survey ${ }^{2}$ (SDSS, AdelmanMcCarthy et al. 2005) archive, and the Six-degree Field Galaxy Survey $^{3}$ (6dFGS) archive (Jones et al. 2004). As the 6dFGS archive provides spectra which are not calibrated in flux, we used the optical photometric information in Jones et al. (2005) and Doyle et al. (2005) to calibrate the 6dFGS data presented here. The only exception was source Swift J0342.0-2115, for which we used the optical photometric information available in SIMBAD $^{4}$ because its $R$ magnitude, essential for the spectrum flux calibration, was not reported in Jones et al. (2005).

\footnotetext{
${ }^{1}$ IRAF is the Image Reduction and Analysis Facility made available to the astronomical community by the National Optical Astronomy Observatories, which are operated by AURA, Inc., under contract with the US National Science Foundation. It is available at http://iraf. noao.edu/

2 http://www.sdss.org

${ }^{3}$ http://www.aao.gov.au/local/www/6df/

${ }_{4}$ available at http://simbad.u-strasbg.fr/simbad/
} 
The identification and classification approach we adopt in the analysis of the optical spectra is the following: for the emissionline AGN classification, we used the criteria of Veilleux \& Osterbrock (1987) and the line ratio diagnostics of Ho et al. (1993, 1997) and of Kauffmann et al. (2003); for the subclass assignation to Seyfert 1 galaxies, we used the $\mathrm{H}_{\beta} /[\mathrm{O}$ III $] \lambda 5007$ line flux ratio criterion as in Winkler et al. (1992).

When possible, in order to provide an estimate of the local absorption in the Seyfert 2 galaxies of our sample and an assessment of the Compton nature of these AGNs, we first dereddened the $\mathrm{H}_{\alpha}$ and $\mathrm{H}_{\beta}$ line fluxes by applying a correction for the Galactic absorption along the line of sight of the source. This was possible by using the estimate of the Galactic color excess $E(B-V)_{\text {Gal }}$ given by Schlegel et al. (1998), and the Galactic extinction law by Cardelli et al. (1989). Then, we estimated the color excess $E(B-V)_{\mathrm{AGN}}$ local to the AGN host from the comparison between the intrinsic line ratio and that corrected for Galactic reddening, using again the extinction law of Cardelli et al. (1989) and assuming an intrinsic $\mathrm{H}_{\alpha} / \mathrm{H}_{\beta}$ line ratio of 2.86 (Osterbrock 1989).

The spectra of these objects are not corrected for starlight contamination (see, e.g., Ho et al. 1993, 1997), because of their limited $\mathrm{S} / \mathrm{N}$ and the spectral resolution. This however does not affect our results and conclusions. In this work we consider a cosmology with $H_{0}=65 \mathrm{~km} \mathrm{~s}^{-1} \mathrm{Mpc}^{-1}, \Omega_{\Lambda}=0.7$ and $\Omega_{\mathrm{m}}=$ 0.3 ; the luminosity distances of the objects reported in this paper are computed from these parameters using the Cosmology Calculator of Wright (2006).

In Figs. 1 and 2 we show the optical finding charts of the 17 sources presented in this work. The corresponding optical counterparts are indicated with tick marks. In Table 1 the detailed log of observations is reported.

We list in Col. 1 the name of the observed Swift sources. In Cols. 2 and 3 we report the coordinates of the objects obtained from the 2MASS catalog ${ }^{5}$ (Skrutskie et al. 2006). In Col. 4 we list the telescope and the instrument used for the source observation. The characteristics of each spectrograph are given in Cols. 5 and 6. Column 7 provides the observation date and the UT time at mid-exposure, while Col. 8 reports the exposure times and the number of spectral pointings.

\section{X-ray data analysis}

Here we report the X-ray data analysis of the 17 sources identified in this work. It is worth noting that for most of the sources the X-ray spectral parameters $\left(\Gamma, N_{\mathrm{H}}\right.$ and possibly the $2-10 \mathrm{keV}$ flux) were already published by various authors; here for consistency we decided to re-analyze the X-ray data in order to always apply the same simple model (an absorbed power law) and to properly use the values obtained in the diagnostic diagram of Malizia et al. (2007 see Sect. 4.19). For 13 out of 17 objects, data acquired with the XRT were analyzed, while for the remain archival XMM-Newton and Chandra observations have been considered.

For Swift J0739.6-3144 XMM-Newton EPIC-pn (Struder et al. 2001) data were analyzed (see Sect. 4.9) while for Swift J0743.0-2543 an XMM-Newton slew pointing is available and therefore only the $2-10 \mathrm{keV}$ flux value can be used. The two remaining sources, Swift J0727.5-2406 and Swift J0811.5+0937, were observed with the Chandra X-ray Observatory for $5126 \mathrm{~s}$ (start time 07:49:36 UT of 2007-12-20) and 5139 s (start time 15:04:24 UT of 2007-12-27), respectively, but since both the

5 available at http://www.ipac.caltech.edu/2mass/ sources are very dim, we were only able to estimate their 2$10 \mathrm{keV}$ fluxes using the eff 2 evt tool of the CIAO Chandra analysis software.

The Chandra data reduction was performed using CIAO v4.1 software with the calibration database CALDB v4.1, provided by the Chandra X-ray Center and following the science threads listed on the CIAO website ${ }^{6}$.

XRT data reduction was performed using the XRTDAS standard data pipeline package (XRTPIPELINE v. 0.12.1) in order to produce screened event files. All data were extracted only in the photon counting (PC) mode (Hill et al. 2004), adopting the standard grade filtering (0-12 for PC) according to the XRT nomenclature. For observations in which the XRT count rate was high enough to produce event pile-up, we extracted the events in an annulus centered on the source, determining the size of the inner circle according to the procedure described in Romano et al. (2006). In the other cases, events for spectral analysis were extracted within a circular region of radius $20^{\prime \prime}$, centered on the source position, which encloses about $90 \%$ of the PSF at $1.5 \mathrm{keV}$ (see Moretti et al. 2004). The background was taken from various source-free regions close to the X-ray source of interest, using circular regions with different radii in order to ensure an evenly sampled background. In all cases, the spectra were extracted from the corresponding event files using the XSELECT software and binned using GRPPHA in an appropriate way, so that the $\chi^{2}$ statistic could be applied. We used version v.011 of the response matrices and create individual ancillary response files using XRTMKARF V. 0.5.6.

XMM-Newton EPIC-pn (Struder et al. 2001) data reduction was performed using the $X M M$ standard analysis software (SAS) version 8.0 employing the latest available calibration files. Only patterns corresponding to single and double events (PATTERN $\leq 4)$ were taken into account; the standard selection filter FLAG $=0$ was applied. The observations were filtered for periods of high background. Source counts were extracted from a circular region of radius $25^{\prime \prime}$ centred on the source in order to exclude the extended emission associated with the galaxy; background spectra were extracted from circular regions close to the source, or from source-free regions of $80^{\prime \prime}$ radius. The ancillary response matrices and the detector response matrices were generated using the XMM SAS tasks arfgen and rmfgen; spectral channels were rebinned in order to achieve a minimum of 20 counts for each bin.

Spectral analyses were performed using XSPEC version 12.5 .0 and all errors are quoted at $90 \%$ confidence level for one parameter of interest $\left(\Delta \chi^{2}=2.71\right)$. For sources with more than one pointing, we performed the spectral analysis of the combined spectra in order to improve the statistical quality of the data. Due to the low statistics available, we identified for each source the best energy range for the spectral analysis and we employed a simple power law (often fixing the photon index to a canonical value of 1.8), absorbed by both the Galactic (Dickey \& Lockman 1990) and an intrinsic neutral hydrogen column density as our baseline model.

\section{Results}

In this section we describe the results of the observations reported in the previous sections. The $B$ magnitudes and the redshifts of the objects mentioned below, if not otherwise stated, are extracted from the LEDA archive (Prugniel et al. 2005). Moreover, for each source we list X-ray, radio and Infrared

\footnotetext{
${ }^{6}$ Available at http://cxc. harvard. edu/ciao/
} 


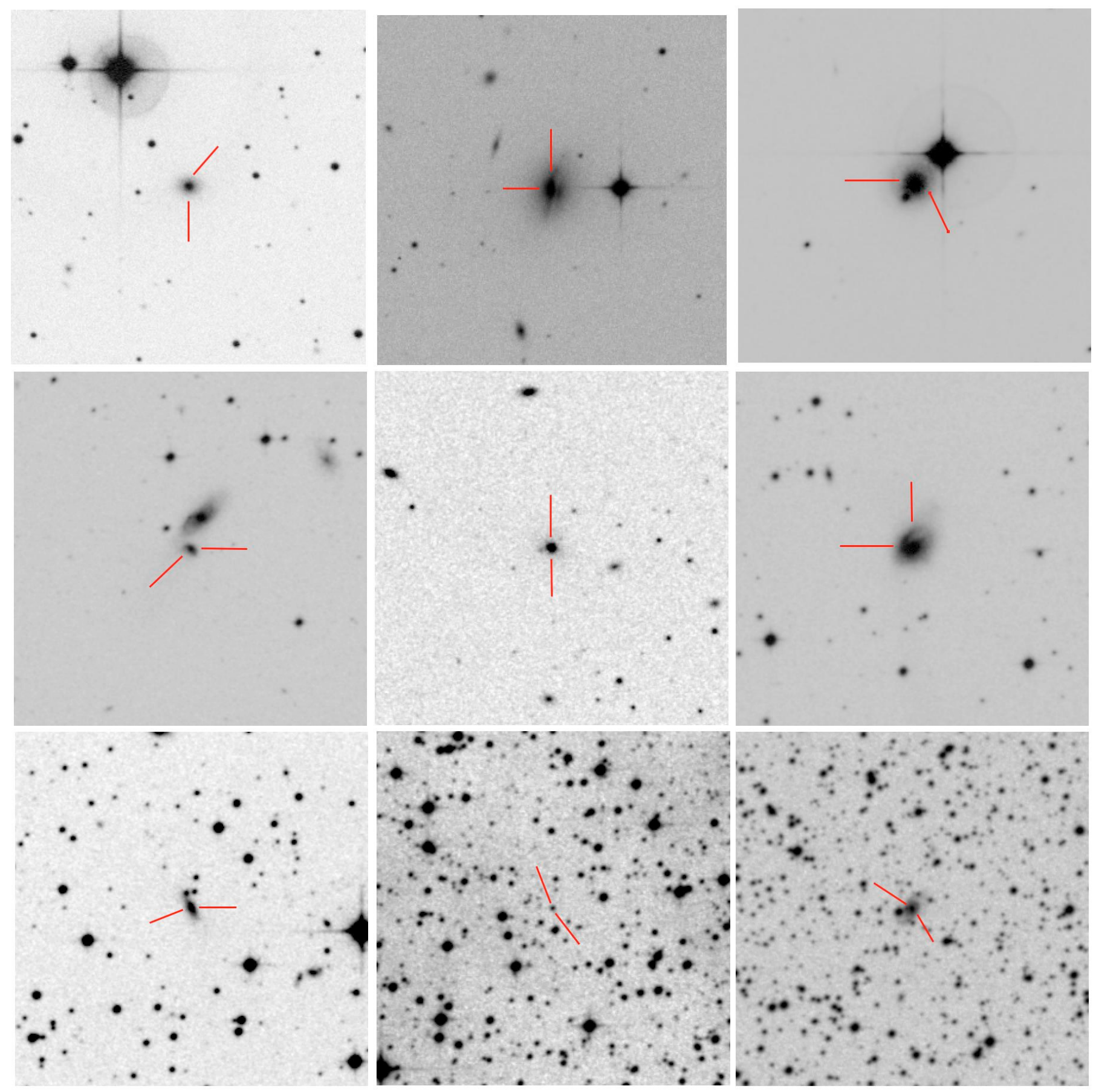

Fig. 1. From left to right and top to bottom: optical images of the fields of Swift J0059.4+3150, Swift J0134.1-3625, Swift J0342.0-2115, Swift J0350.1-5019, Swift J0505.7-2348, Swift J0501.9-3239, Swift J0640.1-4328, Swift J0727.5-2406 and Swift J0739.6-3144. The optical counterparts of the Swift sources are indicated with tick marks. Field sizes are $5^{\prime} \times 5^{\prime}$ and are extracted from the DSS-II-Red survey. In all cases, North is up and East to the left.

counterparts in the ROSAT all sky survey bright source Catalog (Voges et al. 1999), RXTE slew survey (Revnivtsev et al. 2004), XMM-Newton serendipitous survey (Watson et al. 2009), NVSS radio Catalog (Condon et al. 1998), SUMSS Catalog (Mauch et al. 2003), MGPS-2 catalog (Murphy et al. 2007) and IRAS point source Catalog (IRAS 1988).

The optical analysis of the 17 extragalactic objects in our sample reveal that 16 are AGNs and 1 is an X-ray bright optically normal galaxy (XBONG), that is a "passive" galaxy with absorption lines in the optical spectrum (Comastri et al. 2002). The results of our optical study are reported in Table 2 where we list for each source the $\mathrm{H}_{\alpha}, \mathrm{H}_{\text {beta }}$ and [OIII] fluxes, the classification, the redshift estimated from the narrow lines, the luminosity distance given in $\mathrm{Mpc}$, the Galactic color excess and the color excess local to the AGN host and finally the radio luminosity.

The results of the X-ray analysis are reported in Table 3 where we list the X-ray coordinates, the values of the spectral parameters obtained together with the values of the Galactic column density, the hard X-ray (14-195 keV) flux collected from the literature and the luminosity in the energy ranges $2-10 \mathrm{keV}$ and $14-195 \mathrm{keV}$. We point out that generally the values of the spectral parameters found with our analysis are in good 

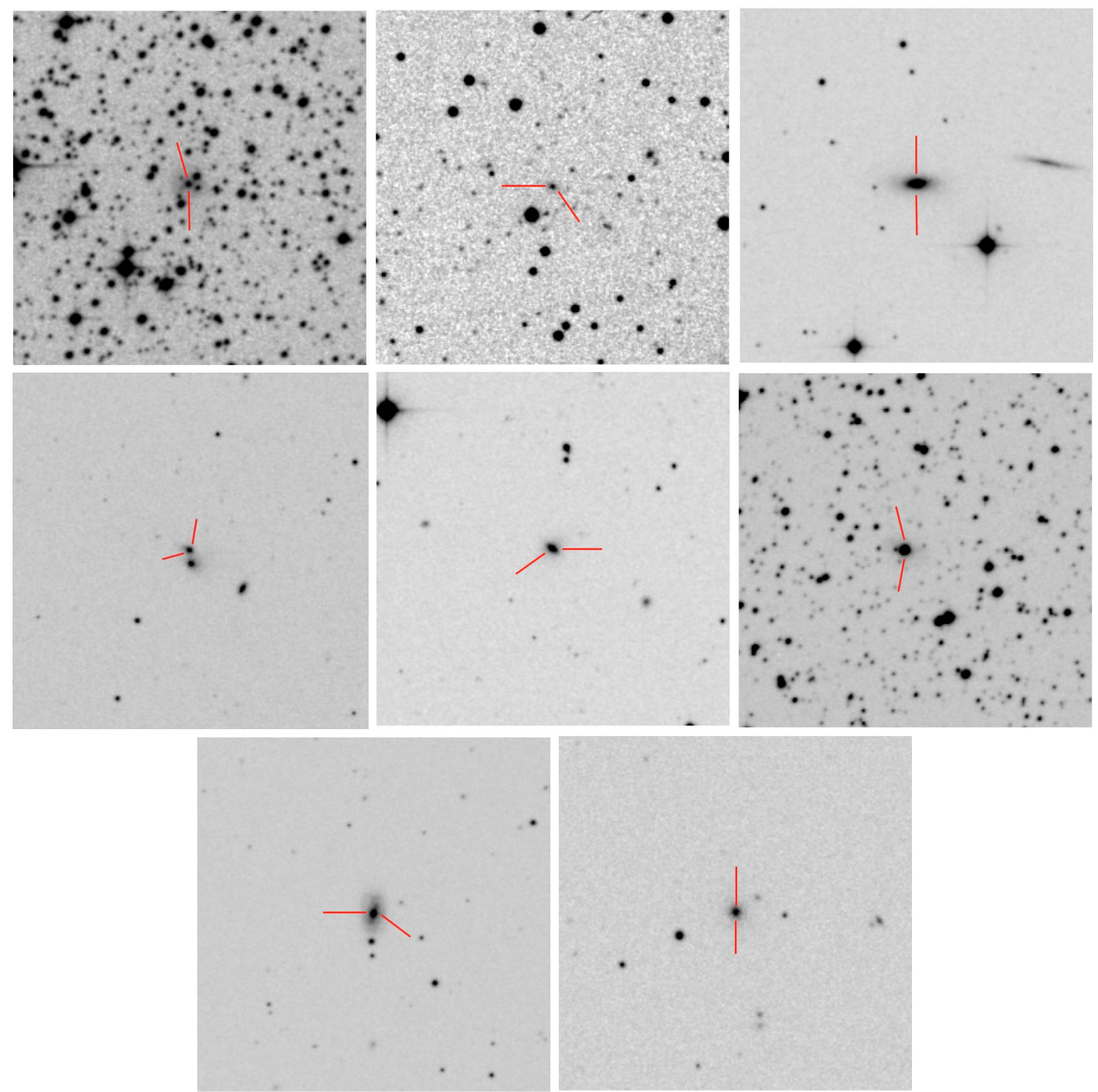

Fig. 2. As Fig. 1, for the fields of Swift J0743.0-2543, Swift J0811.5+0937, Swift J0902.0+6002.Swift J0904.3+5538, Swift J 0911.2+4533, Swift J0917.2-6221, Swift J0923.7+2255 and Swift J1049.4+2258. The optical counterparts of the Swift sources are indicated with tick marks. Field sizes are $5^{\prime} \times 5^{\prime}$ and are extracted from the DSS-II-Red survey. In all cases, North is up and East to the left.

agreement with those reported before (e.g. Tueller et al. 2009; Winter et al. 2008). We would like to point out that all previous classifications of our sample sources reported in the literature are not supported by any published optical spectrum. Only one source, Swift J0505.7-2348, has a published spectrum, which is however normalized to the continuum level optical spectrum (Bikmaev et al. 2006): in this work we have performed a deeper study on the source using a flux-calibrated optical spectrum.

Below we present the main results on the objects of our sample.

\subsection{Swift J0059.4+3150}

This source, identified as Mrk 352, is associated with the galaxy LEDA 3575 with magnitude $B=14.93$ and redshift of 0.0149 . It is associated also with the ROSAT bright source 1RXS J005953.3+314934, the XMM-Newton serendipitous source 2XMM J005953.2+314937 and with the IRAS point source IRAS $00572+3134$.

Here, we publish for the first time an optical spectrum of Swift J0059.4+3150 (Fig. 3, upper left panel). It shows a weak continuum, with broad $\mathrm{H}_{\alpha}+[\mathrm{NII}]$ complex in emission, $\mathrm{H}_{\beta}$ and $\mathrm{H}_{\gamma}$ emission lines, and the [OIII] $\lambda 5007$ forbidden narrow emission line. These allow us to derive the redshift of $0.015 \pm 0.001$, consistent with the redshift determination reported in the Hyperleda archive. Thus, we refine the Seyfert 1 AGN classification reported by Tueller et al. (2008) to Seyfert 1.2.

\subsection{Swift 0134.1-3625}

This object is associated with the galaxy LEDA 5827 with a magnitude $B=14.04$ and a redshift 0.0299 . Moreover, it is listed in the 2nd $X M M$ serendipitous source catalog as $2 \mathrm{XMMi}$ J013357.6-362935 and in the IRAS Point Source Catalog as IRAS 03398-2124. 
Table 2. Main results obtained from the analysis of the optical spectra of the 17 AGNs of the present sample of Swift sources.

\begin{tabular}{|c|c|c|c|c|c|c|c|c|c|}
\hline \multirow[t]{2}{*}{ Object } & \multirow[t]{2}{*}{$\overline{F_{\mathrm{H}_{\alpha}}}$} & \multirow[t]{2}{*}{$\overline{F_{\mathrm{H}_{\beta}}}$} & \multirow[t]{2}{*}{ 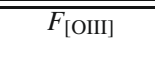 } & \multirow[t]{2}{*}{ Class } & \multirow[t]{2}{*}{$\bar{z}$} & \multirow{2}{*}{$\begin{array}{c}D_{L} \\
(\mathrm{Mpc}) \\
\end{array}$} & \multicolumn{2}{|c|}{$E(B-V)$} & \multirow{2}{*}{$\begin{array}{c}L_{\text {Radio }} \\
\left(\mathrm{erg} \mathrm{s}^{-1}\right)\end{array}$} \\
\hline & & & & & & & Gal. & AGN & \\
\hline Swift J0059.4+3150 & * & $\begin{array}{c}87 \pm 5 \\
{[105 \pm 6]}\end{array}$ & $\begin{array}{c}37 \pm 2 \\
{[45 \pm 3]}\end{array}$ & Sy1.2 & 0.015 & 84.2 & 0.061 & - & - \\
\hline Swift J0134.1-3625 & $\begin{array}{c}9.4 \pm 0.3 \\
{[9.9 \pm 0.4]}\end{array}$ & $\begin{array}{l}\text { in abs. } \\
\text { [in abs.] }\end{array}$ & $\begin{array}{c}5.3 \pm 2.2 \\
{[5.6 \pm 2.3]}\end{array}$ & Sy2 & 0.029 & 136.7 & 0.020 & - & - \\
\hline Swift J0342.0-2115 & * & $\begin{array}{c}2000 \pm 400 \\
{[2700 \pm 600]}\end{array}$ & $\begin{array}{c}400 \pm 80 \\
{[460 \pm 90]}\end{array}$ & Sy1 & 0.0139 & 67.6 & 0.032 & - & - \\
\hline Swift J0350.1-5019 & $\begin{array}{c}38 \pm 3 \\
{[39 \pm 3]}\end{array}$ & $\begin{array}{c}6.9 \pm 0.9 \\
{[7.2 \pm 0.9]}\end{array}$ & $\begin{array}{c}11 \pm 1 \\
{[11 \pm 1]}\end{array}$ & Sy2 & 0.035 & 165.8 & 0.015 & 0.216 & - \\
\hline Swift J0505.7-2348 & $\begin{array}{c}38 \pm 1 \\
{[41 \pm 1]}\end{array}$ & $\begin{array}{c}9 \pm 1 \\
{[10 \pm 1]}\end{array}$ & $\begin{array}{c}48 \pm 1 \\
{[55 \pm 1]}\end{array}$ & Sy2 & 0.036 & 172.1 & 0.040 & 0.144 & $3.1 \times 10^{38}(1.4 \mathrm{GHz})$ \\
\hline Swift J0501.9-3239 & $\begin{array}{l}180 \pm 20 \\
{[190 \pm 20]}\end{array}$ & $\begin{array}{c}52 \pm 11 \\
{[59 \pm 13]}\end{array}$ & $\begin{array}{c}320 \pm 30 \\
{[350 \pm 30]}\end{array}$ & Sy2 & 0.0126 & 58.9 & 0.017 & 0.036 & $7.0 \times 10^{37}(843 \mathrm{MHz})$ \\
\hline Swift J0640.1-4328 & $\begin{array}{c}3.1 \pm 0.5 \\
{[4.1 \pm 0.7]}\end{array}$ & $\begin{array}{c}0.7 \pm 0.3 \\
{[3.6 \pm 1.7]}\end{array}$ & $\begin{array}{c}2.3 \pm 0.4 \\
{[3.0 \pm 0.6]}\end{array}$ & Sy2 & 0.061 & 293.9 & 0.084 & - & $8.1 \times 10^{39}(843 \mathrm{MHz})$ \\
\hline Swift J0727.5-2406 & * & $\begin{array}{l}2.1 \pm 0.7 \\
{[47 \pm 7]}\end{array}$ & $\begin{array}{c}21 \pm 1 \\
{[385 \pm 17]}\end{array}$ & Sy1.9 & 0.123 & 619.0 & 1.047 & - & $1.9 \times 10^{40}(1.4 \mathrm{GHz})$ \\
\hline Swift J0739.6-3144 & $\begin{array}{c}36 \pm 2 \\
142 \pm 7\end{array}$ & $\begin{array}{c}2.3 \pm 0.5 \\
{[18 \pm 4]}\end{array}$ & $\begin{array}{c}32 \pm 1 \\
{[216 \pm 5]}\end{array}$ & Sy2 & 0.026 & 122.3 & 0.613 & 0.360 & $\begin{array}{l}2.5 \times 10^{38}(1.4 \mathrm{GHz}) \\
6.4 \times 10^{38}(843 \mathrm{MHz})\end{array}$ \\
\hline Swift J0743.0-2543 & * & $\begin{array}{c}11 \pm 2 \\
{[146 \pm 20]}\end{array}$ & $\begin{array}{c}4.5 \pm 0.7 \\
{[38 \pm 6]}\end{array}$ & Sy 1.2 & 0.023 & 108.0 & 0.678 & - & $1.2 \times 10^{37}(1.4 \mathrm{GHz})$ \\
\hline Swift J0811.5+0937 & - & $\begin{array}{l}\text { in abs. } \\
\text { [in abs.] }\end{array}$ & - & XBONG & 0.286 & 1582.6 & 0.026 & - & $1.8 \times 10^{40}(1.4 \mathrm{GHz})$ \\
\hline Swift J0902.0+6007 & * & $\begin{array}{c}52 \pm 5 \\
{[58 \pm 6]}\end{array}$ & $\begin{array}{c}98 \pm 5 \\
{[114 \pm 6]}\end{array}$ & Sy2 & 0.012 & 56.2 & 0.043 & 0.198 & $1.6 \times 10^{38}(1.4 \mathrm{GHz})$ \\
\hline Swift J0904.3+5538 & * & $\begin{array}{c}30.9 \pm 7.9 \\
{[36.1 \pm 9.9]}\end{array}$ & $\begin{array}{c}23.1 \pm 0.8 \\
{[24.5 \pm 0.9]}\end{array}$ & Sy 1.5 & 0.0374 & 177.4 & 0.021 & - & - \\
\hline Swift J0911.2+4533 & $\begin{array}{c}4.2 \pm 0.2 \\
{[4.4 \pm 0.2]}\end{array}$ & $\begin{array}{l}<0.3 \\
{[<0.2]}\end{array}$ & $\begin{array}{c}4.4 \pm 0.2 \\
{[4.4 \pm 0.3]}\end{array}$ & Sy2 & 0.0269 & 127.1 & 0.019 & $>0.360$ & $8.4 \times 10^{37}(1.4 \mathrm{GHz})$ \\
\hline Swift J0917.2-6221 & * & $\begin{array}{c}1450 \pm 40 \\
{[2380 \pm 60]}\end{array}$ & $\begin{array}{c}270 \pm 3 \\
{[518 \pm 6]}\end{array}$ & Sy 1.2 & 0.057 & 274.3 & 0.182 & - & $4.7 \times 10^{39}(843 \mathrm{MHz})$ \\
\hline Swift J0923.7+2255 & * & $\begin{array}{c}127 \pm 13 \\
{[140 \pm 14]}\end{array}$ & $\begin{array}{c}92 \pm 4 \\
{[105 \pm 5]}\end{array}$ & NLSy1 & 0.034 & 160.9 & 0.043 & - & $4.5 \times 10^{38}(1.4 \mathrm{GHz})$ \\
\hline Swift J1049.4+2258 & $\begin{array}{c}26 \pm 1 \\
{[29 \pm 1]}\end{array}$ & $\begin{array}{c}4.4 \pm 0.4 \\
{[4.7 \pm 0.5]}\end{array}$ & $\begin{array}{c}87 \pm 1 \\
{[93 \pm 1]}\end{array}$ & Sy2 & 0.033 & 156.0 & 0.029 & 0.261 & - \\
\hline
\end{tabular}

Note: emission line fluxes are reported both as observed and (between square brackets) corrected for the intervening Galactic absorption $E(B-V)_{\mathrm{Gal}}$ along the object line of sight (from Schlegel et al. 1998). Line fluxes are in units of $10^{-15} \mathrm{erg} \mathrm{cm}^{-2} \mathrm{~s}^{-1}$. The typical error on the redshift measurement is \pm 0.001 but for the SDSS and 6dFGS spectra, for which an uncertainty of \pm 0.0003 can be assumed; *: heavily blended with [N II] lines.

The optical spectrum of this source (Fig. 3, upper right panel) shows a red stellar continuum, with a weak narrow emission $\mathrm{H}_{\alpha}+[\mathrm{NII}]$ complex and a weaker forbidden [OIII] emission line. The lines have a redshift of $0.029 \pm 0.001$, consistent with that reported in the Hyperleda archive. We classify for the first time this source as a Seyfert 2 galaxy. Our classification is more thorough with respect to that given by SIMBAD (in which the source is reported as "radio galaxy") and in Winter et al. (2008) who reported it as "galaxy".

\subsection{Swift J0342.0-2115}

This Swift source is associated with the galaxy LEDA 13590 with magnitude $B=13.31$ and a redshift of 0.0145 . It is associated with a ROSAT bright source, an XMM serendipitous source (1RXS J034203.8-211428 and 2XMM J034203.6-211439 respectively) and with an IRAS point source, IRAS 03398-2124.

Its optical spectrum (Fig. 3, central left panel) shows the emission $\mathrm{H}_{\alpha}+[\mathrm{NII}]$ complex, with the presence of the broad emission $\mathrm{H}_{\beta}$ as well as the narrow [OIII] forbidden emission 
Table 3. Main results obtained from the analysis of the X-ray spectra of the 17 sources of the present sample.

\begin{tabular}{|c|c|c|c|c|c|c|c|c|c|}
\hline Source & RA & Dec & $\begin{array}{c}N_{\mathrm{H}} \mathrm{Gal} \\
\times 10^{22} \mathrm{~cm}^{-2} \\
\end{array}$ & $\begin{array}{c}N_{\mathrm{H}}^{\bullet} \\
\times 10^{22} \mathrm{~cm}^{-2} \\
\end{array}$ & $\Gamma^{+}$ & $\begin{array}{c}F_{(2-10) \mathrm{keV}} \\
\times 10^{-12} \mathrm{erg} \mathrm{s}^{-1} \mathrm{~cm}^{-2} \\
\end{array}$ & $\begin{array}{c}F_{(14-195) \mathrm{keV}} \\
\times 10^{-11} \mathrm{erg} \mathrm{s}^{-1} \mathrm{~cm}^{-2} \\
\end{array}$ & $\begin{array}{c}L_{\mathrm{X}} \\
10^{43} \mathrm{erg} \mathrm{s}^{-1} \\
\end{array}$ & Ref.* \\
\hline Swift J0059.4+3150 & 005953.29 & +314936.6 & 0.055 & - & $1.67_{-0.03}^{+0.03}$ & 7.3 & 4.2 & $\begin{array}{c}0.6(2-10) \\
3.1(14-195)\end{array}$ & 1 \\
\hline Swift J0134.1-3625 & 013357.71 & -362938.4 & 0.019 & $75_{-21}^{+34}$ & {$[1.8]$} & 1.9 & 5.4 & $\begin{array}{c}0.8(2-10) \\
7.2(14-195)\end{array}$ & 1 \\
\hline Swift J0342.0-2115 & 034203.62 & -211439.6 & 0.023 & - & $1.94_{-0.05}^{+0.05}$ & 23.8 & 4.6 & $\begin{array}{c}\sim 1.3(2-10) \\
1.8(14-195)\end{array}$ & 1 \\
\hline Swift J0350.1-5019 & 035023.08 & -501811.4 & 0.012 & $17_{-7}^{+9}$ & {$[1.8]$} & 3.1 & 3.1 & $\begin{array}{c}1.0(2-10) \\
9.5(14-195)\end{array}$ & 1 \\
\hline Swift J0505.7-2348 & 050545.70 & -235113.3 & 0.021 & $6_{-0.5}^{+0.5}$ & $1.4_{+0.3}^{-0.4}$ & 15.5 & 7.2 & $\begin{array}{c}5.3(2-10) \\
21.6(14-195)\end{array}$ & 1 \\
\hline Swift J0501.9-3239 ${ }^{A}$ & 051935.82 & -323926.6 & 0.018 & - & $1.45_{-0.05}^{+0.05}$ & 13.6 & 6.2 & $\begin{array}{c}0.5(2-10) \\
1.2(14-195)\end{array}$ & 1 \\
\hline Swift J0640.1-4328 ${ }^{A}$ & 064037.89 & -432119.8 & 0.025 & $11.7_{-3.6}^{+4.5}$ & {$[1.8]$} & 2.2 & 2.0 & $\begin{array}{c}2.3(2-10) \\
28.9(14-195)\end{array}$ & 1 \\
\hline Swift J0727.5-2406 ${ }^{C}$ & 072721.05 & -240632.2 & 0.650 & - & - & 0.6 & 2.2 & $\begin{array}{c}2.7(2-10) \\
93.2(14-195)\end{array}$ & 2 \\
\hline Swift J0739.6-3144 ${ }^{B}$ & 073944.61 & -314301.6 & 0.431 & $\begin{array}{l}47_{-12}^{+13},[0.7] \\
14_{-1.9}^{+2.3},[0.9]\end{array}$ & $1.77_{-0.09}^{+0.07}$ & 0.9 & 2.5 & $\begin{array}{c}0.2(2-10) \\
4.5(14-195)\end{array}$ & 2 \\
\hline Swift J0743.0-2543 ${ }^{B}$ & 074314.95 & -254544.3 & 0.581 & - & - & $14.7^{\Delta}$ & 2.0 & $\begin{array}{c}2.1(2-10) \\
2.8(14-195)\end{array}$ & 2 \\
\hline Swift J0811.5+0937 ${ }^{C}$ & 081130.99 & +093351.6 & 0.024 & - & - & 0.1 & 2.6 & $\begin{array}{c}3.0(2-10) \\
778.1(14-195)\end{array}$ & 2 \\
\hline Swift J0902.0+6007 ${ }^{A}$ & 090158.81 & +600905.8 & 0.043 & $8_{-4.0}^{+8.6}$ & {$[1.8]$} & 1.1 & 3.1 & $\begin{array}{c}0.04(2-10) \\
1.2(14-195)\end{array}$ & 1 \\
\hline Swift J0904.3+5538 & 090436.92 & +5535 59.7 & 0.022 & - & $1.44_{-0.07}^{+0.07}$ & 4.9 & 1.9 & $\begin{array}{c}1.8(2-10) \\
7.2(14-195)\end{array}$ & 1 \\
\hline Swift J0911.2+4533 & 091129.98 & +452804.1 & 0.012 & $30_{-7}^{+9.5}$ & [1.8] & 2.0 & 1.8 & $\begin{array}{c}0.4(2-10) \\
5.8(14-195)\end{array}$ & 1 \\
\hline Swift J0917.2-6221 ${ }^{A}$ & 091609.14 & -621928.4 & 0.158 & $0.7_{-0.3}^{+0.3}$ & $1.67_{-0.2}^{+0.2}$ & 16.0 & 3.3 & $\begin{array}{c}14.8(2-10) \\
28.8(14-195)\end{array}$ & 1 \\
\hline Swift J0923.7+2255 & 092342.89 & +225433.2 & 0.031 & - & $1.95_{-0.05}^{+0.05}$ & 11.8 & 4.5 & $\begin{array}{c}3.7(2-10) \\
12.7(14-195)\end{array}$ & 1 \\
\hline Swift J1049.4+2258 ${ }^{A}$ & 104930.88 & +225752.9 & 0.019 & $20_{-3}^{+5}$ & {$[1.8]$} & 0.7 & 3.7 & $\begin{array}{c}0.2(2-10) \\
10.9(14-195)\end{array}$ & 1 \\
\hline
\end{tabular}

+ The square brackets in the $\Gamma$ column indicate that we used a fixed value; ${ }^{*}$ this column reports the references for the hard $\mathrm{X}$-ray flux; ${ }^{\Delta}$ source fluxes were calculated from count rates (see http://heasarc.gsfc.nasa.gov/db-perl/W3Browse/w3table.pl); ${ }^{\bullet}$ the values in the square brackets indicate the covering fraction; ${ }^{1}$ Tueller et al. (2009); ${ }^{2}$ Ajello et al. (2007); ${ }^{A}$ soft X-ray data from Swift/XRT; ${ }^{B}$ soft X-ray data from $X M M /$ EPIC; ${ }^{C}$ soft X-ray data from Chandra.

lines. These lines allow us to derive a redshift of $0.0139 \pm 0.0003$, somewhat in agreement with that reported in the Hyperleda archive. Here, we classify for the first time this source as a Seyfert 1 AGN.

\subsection{Swift J0350.1-5019}

The optical counterpart of this Swift source is the galaxy LEDA 13946 with magnitude $B=16.19$.

Its optical spectrum (Fig. 3, central right panel) shows a weak continuum with obvious emission lines; a narrow $\mathrm{H}_{\alpha}+[\mathrm{NII}]$ complex, $\mathrm{H}_{\beta}$ and $\mathrm{H}_{\gamma}$ permitted narrow lines,
[OI] $\lambda 6300,[\mathrm{OIII}] \lambda 5007$ and $[\mathrm{OII}] \lambda 3727$ forbidden narrow lines. From the features above we are able to classify this source for the first time as a Seyfert $2 \mathrm{AGN}$ at redshift $z=0.035 \pm 0.001$. Our classification is more thorough with respect to that of Tueller et al. (2008) who report it as a "galaxy".

\subsection{Swift J0505.7-2348}

This source is associated with the galaxy LEDA 178130, with magnitude $B=16.76$ and redshift of 0.035 . It is also associated with a NVSS radio source (NVSS 050545-235114) with a flux of $7.9 \pm 0.5 \mathrm{mJy}$ at $1.4 \mathrm{GHz}$. 

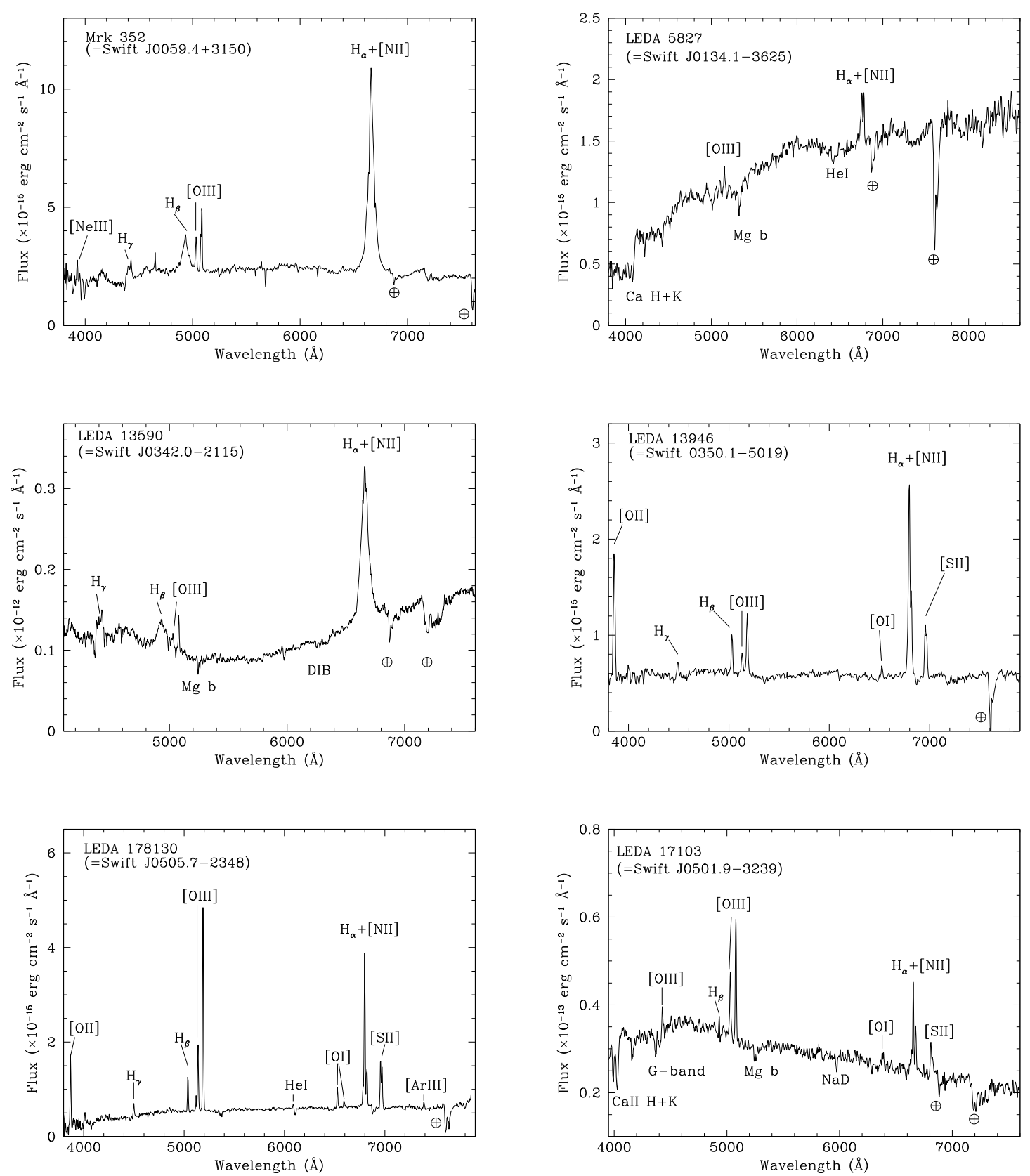

Fig. 3. Spectra (not corrected for the intervening Galactic absorption) of the optical counterpart of Swift J0059.4+3150, Swift J0134.1-3625, Swift J0342.0-2115, Swift J0350.1-5019, Swift J0505.7-2348 and Swift J0501.9-3239.

Its spectrum (Fig. 3, bottom left panel) shows a very narrow emission $\mathrm{H}_{\alpha}+[\mathrm{NII}]$ complex with $\mathrm{H}_{\beta}$ and $\mathrm{H}_{\gamma}$ permitted narrow emission lines, [OI] $\lambda 6300$, [OIII] $] \lambda 5007$ and [OII] $\lambda 3727$ forbidden narrow emission lines. Therefore, we classify this Swift source as a Seyfert 2 AGN with $z=0.036 \pm 0.001$. Our results are in agreement with those given by Bikmaev et al. (2006).

\subsection{Swift J0501.9-3239}

This object is associated with the galaxy LEDA 17103, with magnitude $B=13.87$ and redshift 0.012337. Listed in the ROSAT Bright Source Catalogue as 1RXS J051936.1323910 , this object has a radio counterpart in the NVSS
(NVSS 051935-323929) and SUMSS catalogues, with density fluxes at $1.4 \mathrm{GHz}$ and $843 \mathrm{MHz}$ of $14.2 \pm 1.0 \mathrm{mJy}$ and $20.1 \pm$ $2.0 \mathrm{mJy}$, respectively. It also shows an infrared counterpart in the IRAS Point Source Catalog named as IRAS 05177-3242.

As can be see in Fig. 3 (lower right panel), its optical spectrum shows narrow $\mathrm{H}_{\alpha}$ and $\mathrm{H}_{\beta}$ lines in emission, as well as [O III] forbidden emission line. CaII $H+K$ and G-band are also present in absorption. From the features listed above, we find that Swift J0501.9-3239 is a Seyfert 2 AGN with $z=$ $0.0126 \pm 0.0003$, in agreement with the redshift reported in the Hyperleda archive. Our classification is thus at variance with that reported in SIMBAD (Seyfert 1 AGN), in Winter et al. (2008) and, who classify it as Seyfert 1.5 AGN. The Swift/XRT 

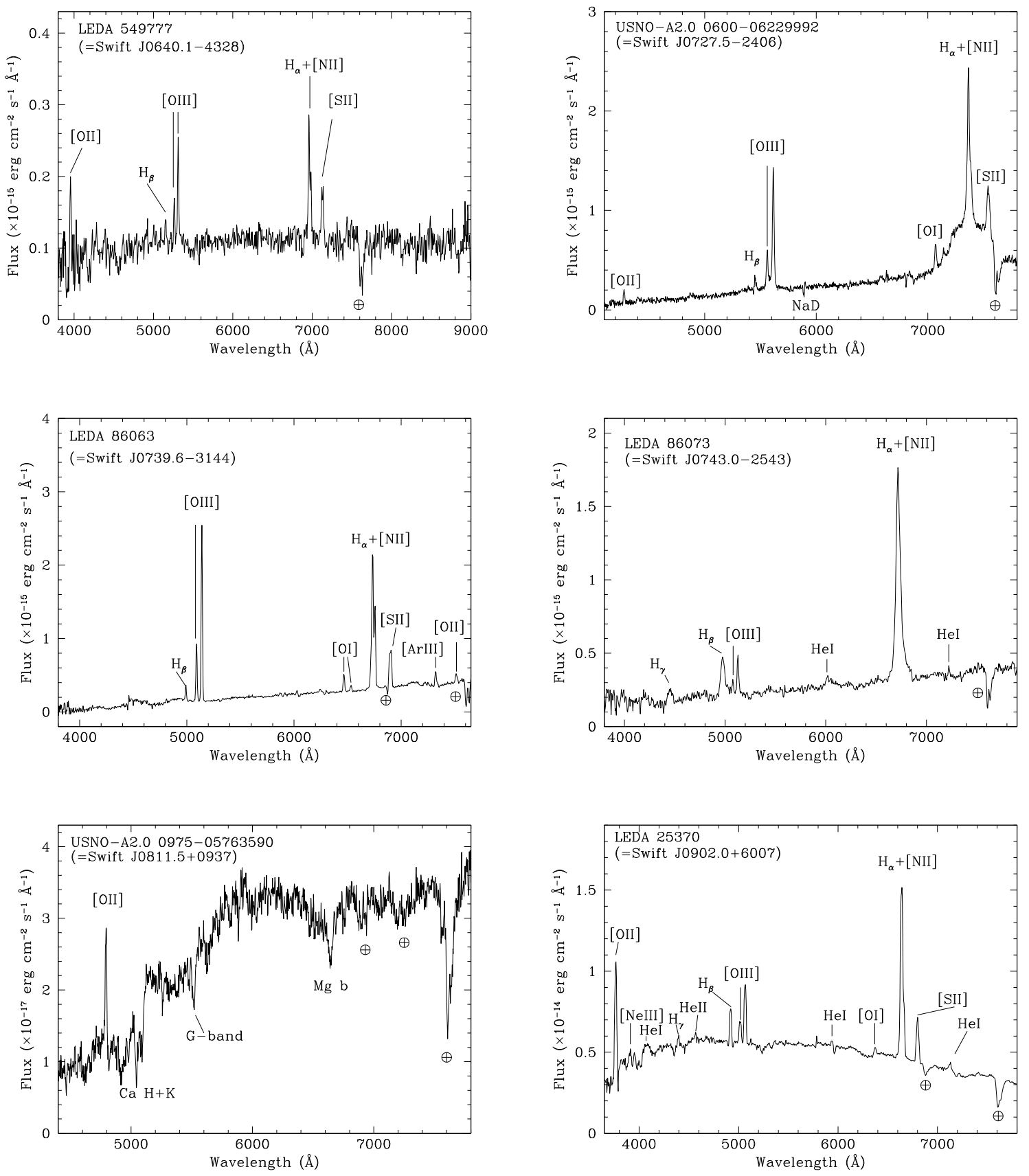

Fig. 4. Spectra (not corrected for the intervening Galactic absorption) of the optical counterpart of Swift J0640.1-4328, Swift J0727.5-2406, Swift J0739.6-3144, Swift J0743.0-2543 and Swift J0811.5+0937, Swift J0902.0+6007.

analysis revealed that this source is a naked Seyfert 2 AGN (see Table 3), that is, a Seyfert 2 with no intrinsic absorption (e.g. Bianchi et al. 2008) in agreement with the optical determination of $\mathrm{E}(B-V)_{\mathrm{AGN}}$ (see Table 2) which is substantially lower than the other type 2 AGNs of our sample.

\subsection{Swift J0640.1-4328}

The optical counterpart of this source has been identified as LEDA 549777, with a $B$ magnitude of 16.79 . This object is also a radio emitter, being reported in the SUMSS catalog with a flux of $93.4 \pm 3.0 \mathrm{mJy}$ at $843 \mathrm{MHz}$.
The optical spectrum (Fig. 4, upper left panel) shows a weak continuum with a low $\mathrm{S} / \mathrm{N}, \mathrm{H}_{\alpha}+[\mathrm{NII}]$ emission complex, a weak narrow $\mathrm{H}_{\beta}$ and [OIII] $\lambda 5007$ forbidden emission line. Using the information extracted from our optical spectrum we are able to classify this object as a Seyfert 2 AGN with a redshift of $0.061 \pm$ 0.001. In the Tueller et al. (2008) catalog this object is classified as "galaxy", with no reported redshift.

\subsection{Swift J0727.5-2406}

The optical counterpart of this Swift source is USNO-A2.0 0600-06229992 with magnitude $R=17.3$. This object, as reported in Ajello et al. (2008), is associated with the ROSAT 
faint source 1RXS J072720.8-240629 and with the radio object NVSS J072721-240632 with a flux density of $29.1 \pm 1.0 \mathrm{mJy}$ at $843 \mathrm{MHz}$.

The optical spectrum (Fig. 4, upper right panel), shows a broad $\mathrm{H}_{\alpha}+[\mathrm{NII}]$ emission complex with narrow $\mathrm{H}_{\beta}$ emission line. Through the $\mathrm{H}_{\beta} /[\mathrm{O}$ III $] \lambda 5007$ line flux ratio we are able to classify for the first time this source as a Seyfert 1.9 AGN with $z=0.123 \pm 0.001$.

\subsection{Swift J0739.6-3144}

Swift J0739.6-3144 is associated with the galaxy LEDA 86063, with magnitude $B=16.51$ and redshift 0.0257 . It is positionally consistent with an NVSS source (NVSS 073944-314304) and a MGPS-2 source, with fluxes of $31.6 \pm 1.1 \mathrm{mJy}$ at $1.4 \mathrm{GHz}$ and $42.4 \pm 2.2 \mathrm{mJy}$ at $843 \mathrm{MHz}$ respectively. The analysis of the optical spectrum (Fig. 4, central left panel), published here for the first time, only shows narrow permitted and forbidden emission lines, which enable us to classify it as a type 2 AGN with redshift $0.026 \pm 0.001$, confirming the classification reported by Ajello et al. (2008).

We now describe in detail the results from the X-ray spectral analysis of this AGN. It is found that a simple absorbed power law fails to reproduce the spectrum of Swift J0739.6$3144\left(\chi^{2}=363\right.$ for 153 d.o.f. $\left.^{7}\right)$ : it also gives a negative value of photon index, no intrinsic absorption and an observed flux of $9.4 \times 10^{-13} \mathrm{erg} \mathrm{cm}^{-2} \mathrm{~s}^{-1}$ in the $2-10 \mathrm{keV}$ band. Inspection of the data residuals obtained using this simple model indicates a presence of strong soft excess below $1 \mathrm{keV}$ and a prominent line around $6.4 \mathrm{keV}$; these features, together with the extremely flat power law, strongly point to a highly absorbed Compton thick AGN.

Following the analysis of IGR J16351-5806, a new highenergy selected Compton thick AGN (Malizia et al. 2009), we tried to fit the soft excess of Swift J0739.6-3144 with the mekal model in XSPEC which fits well the soft part of the spectrum and allows various scenarios (transmission, reflection and complex absorption) to account for the continuum up to $10 \mathrm{keV}$. Although this model yields an adequate fit to our spectrum, it is worth noting that it may be an oversimplified parameterization of the data. High resolution spectroscopy of nearby Seyfert 2s have, in fact, demonstrated that the soft X-ray emission is often dominated by emission lines from photoionized gas which have a negligible contribution from an underlying continuum. The blending of these emission lines in the EPIC spectra can mimic a continuum emission (Iwasawa et al. 2003). However, as our main goal is to study the nature of Swift J0739.6-3144, the uncertainties induced by a purely phenomenological modelling of the soft excess will not substantially affect our results.

The addition of mekal to the absorbed power law improves the fit $\left(\Delta \chi^{2}=79\right.$ for 2 d.o.f.) and gives a gas temperature of $k T$ of $0.65_{-0.07}^{+0.18}$, still leaving an extremely flat power low continuum $(\Gamma \sim 0.6)$. Another substantial improvement $\left(\Delta \chi^{2}=82\right.$ for 3 d.o.f.) is obtained when we introduce the $K \alpha$ iron fluorescence emission line: the energy of the line is found to be at $6.41 \pm 0.04 \mathrm{keV}$ with an equivalent width $E W<1 \mathrm{keV}$. The intrinsic width of the line is narrow $\left(\sigma \sim 0.15_{-0.04}^{+0.07} \mathrm{keV}\right)$ and for simplicity it has been frozen to the observed value in subsequent fits. The flat slope of the power law as well as the strength of the line at $6.4 \mathrm{keV}(E W \sim 0.5-1 \mathrm{keV})$ and the apparent lack of absorption again suggest that the source could be reflection dominated and therefore highly absorbed (Matt et al. 2000).

\footnotetext{
7 d.o.f. $=$ Degrees of freedom
}

The models generally used to account for the X-ray continuum of AGNs in this regime are the transmission model, pure reflection model and, as recently found by Malizia et al. (2009), the complex absorption model (see this latter reference for details). We applied all these models to our data and at first we ruled out the pure reflection one because this model assumes that the absorbed power law is totally absorbed by a column density $N_{\mathrm{H}}>10^{25} \mathrm{~cm}^{-2}$ implying an EW of the $K \alpha$ iron line of $1 \mathrm{keV}$ or even more, while we still measure for our line an equivalent width of less than $1 \mathrm{keV}\left(0.5_{-0.13}^{+0.12} \mathrm{keV}\right)$. Both the transmission and the complex absorption scenario provide a good fit to our data $\left(\chi^{2}=159 / 148\right.$ and $\chi^{2}=159 / 146$ respectively, this implies a chance improvement probability of $\sim 10^{-7}$ for both models according to the F-test, see Press et al. 1992) but while the first still gives a quite flat photon index $(\Gamma=1.25 \pm 0.1)$ with a column density of about $2 \times 10^{23} \mathrm{~cm}^{-2}$, the second provides a more canonical value of the power law continuum of $\left(\Gamma=1.77_{-0.09}^{+0.07}\right)$. The absorption required by the data is in the form of two columns $\left(N_{\mathrm{H}_{1}} \sim 5 \times 10^{23} \mathrm{~cm}^{-2}\right.$ and $N_{\mathrm{H}_{2}} \sim 1.5 \times 10^{23} \mathrm{~cm}^{-2}$ ), both covering $70-90 \%$ of the source. In this case the combinations of such columns are able to explain the observed iron line EW (Ghisellini et al. 1994). In Fig. 6 the unfolded spectrum fitted with this model is shown.

As for the case of IGR J16351-5806, also for Swift J0739.63144 this model is of interest in view of recent studies on the torus geometry and its nature, which strongly indicate that this structure is clumpy and made of dusty clouds (Elitzur 2008). Of course only the study of the broad band spectrum i.e. the combination of the X-ray data with the high energy information $(>10 \mathrm{keV})$ can help to definitely assess the nature of this source i.e. if it is just a highly absorbed object or a definite Compton thick AGN.

\subsection{Swift J0743.0-2543}

This source is associated with the galaxy LEDA 86073, with magnitude $B=14.0$, obtained from the USNO-A2.0 catalog (Monet et al. 2003). It was detected by Ajello et al. (2008), likely associated with a ROSAT all-sky bright source (1RXS J074315.6-254545), with an IRAS source (IRAS 07411-2538) and with a NVSS radio source (NVSS 074314254547 ), with a flux density of $0.203 \pm 0.027 \mathrm{mJy}$ at $1.4 \mathrm{GHz}$.

Its optical spectrum (Fig. 4, central right panel) shows a broad emission $\mathrm{H}_{\alpha}+[\mathrm{NII}]$ complex with broad $\mathrm{H}_{\beta}$ and $\mathrm{HeI}$ emission lines, which enable us to classify for the first time Swift J0743.0-2543 as a Seyfert 1.2 AGN at $z=0.023 \pm 0.001$.

\subsection{Swift J0811.5+0937}

Its optical counterpart is USNO-A2.0 0975-05763590 with a magnitude $B=19.2$ according to the USNO Catalog. It is also associated with an NVSS source (NVSS 081130+093350) with a flux density of $4.3 \pm 0.5 \mathrm{mJy}$ at $1.4 \mathrm{GHz}$. The spectrum (Fig. 4, bottom left panel), shows a forbidden [OII] narrow emission line and absorption features. Following the method of LaurentMuehleisen et al. (1998), for this source we have calculated the Ca II break contrast at $4000 \AA\left(\mathrm{Br}_{4000}\right)$, as defined by Dressler \& Shectman (1987), and its value is $\sim 45 \%$; the presence of other absorption features, such as the $\mathrm{G}$ band, the $\mathrm{Mg}$ I and the $\mathrm{Ca}$ II $\mathrm{H}+\mathrm{K}$ doublet and the lack of strong Balmer absorption lines, enables us to state that this source is a "normal galaxy". This peculiar object is thus an XBONG with redshift of $0.286 \pm 0.001$. Our classification agrees with that reported in Ajello et al. (2008). 

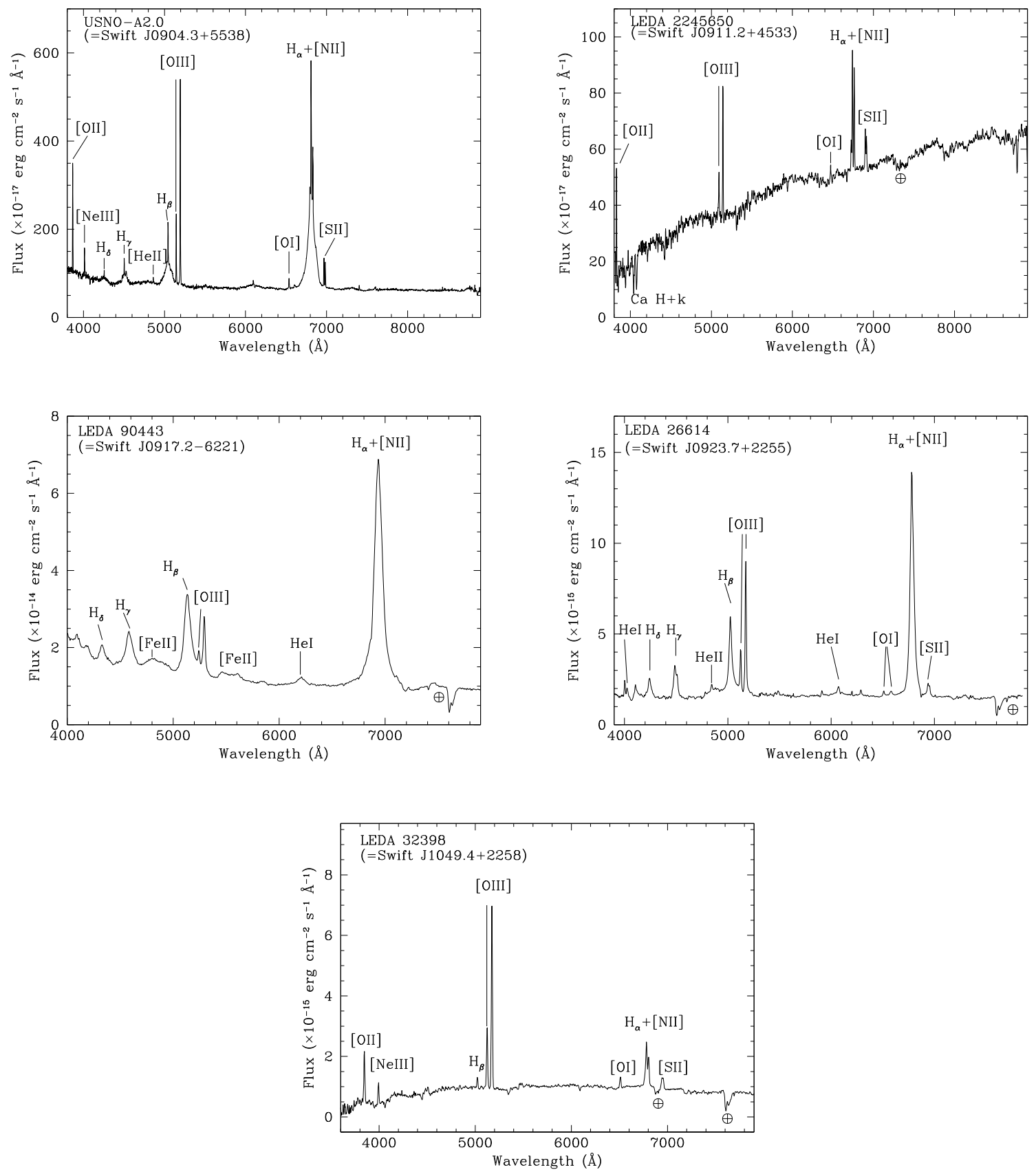

Fig. 5. Spectra (not corrected for the intervening Galactic absorption) of the optical counterpart of Swift J0904.3+5538, Swift J0911.2+4533, Swift J0917.2-6221 and Swift J0923.7+2255, Swift J1049.4+2258.

\subsection{Swift J0902.0+6007}

The optical counterpart is LEDA 25370 with magnitude $B=$ 14.56 and redshfit 0.0111 . This source is associated with an $X M M$ serendipitous source (2XMMi J090158.7+600903) and is listed in the NVSS Catalog (NVSS 090158+600906) with a flux density of $30.3 \pm 1.0 \mathrm{mJy}$ at $1.4 \mathrm{GHz}$.

The optical spectrum (Fig. 4, bottom right panel) shows narrow $\mathrm{H}_{\alpha}+[\mathrm{NII}]$ emission complex, $\mathrm{H}_{\beta}$ and $\mathrm{H}_{\gamma}$ permitted narrow emission lines, weak HeI and HeII emission lines; [OIII] and [OII] forbidden emission lines are also present. Through these lines, we estimate a redshift of $0.012 \pm 0.001$, consistent with that reported in the Hyperleda archive. As before, through the diagnostic diagrams listed in Sect. 2 we can classify the object for the first time as a Seyfert 2 AGN. In the Tueller et al. (2008) catalog, this source was simply classified as galaxy.

\subsection{Swift J0904.3+5538}

The optical counterpart is USNO-A2.0 1425-07270668, with a magnitude $R=13.5$, provided in this same catalog. This Swift source is positionally consistent with an XMM serendipitous source (2XMMi J090436.9+553602).

Its optical spectrum showed in Fig. 5 (upper left panel) has all the typical features of a broad line AGN: a broad emission $\mathrm{H}_{\alpha}+[\mathrm{NII}]$ complex with broad $\mathrm{H}_{\beta}$ and $\mathrm{H}_{\gamma}$ emission lines and 


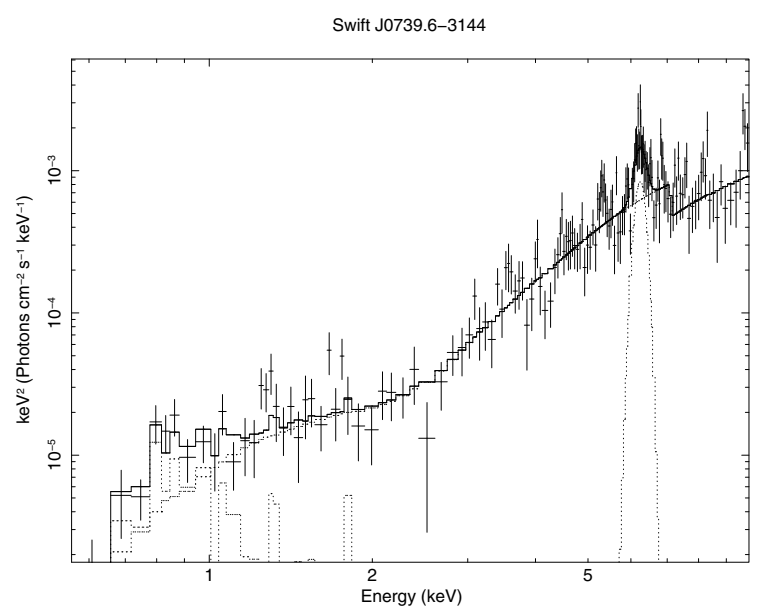

Fig. 6. XMM EPIC-pn X-ray spectrum of Swift J0739.6-3144, possible Compton thick source. The solid line shows the best fit model.

some forbidden narrow emission lines. Through the optical spectrum and the $\mathrm{H}_{\beta} /[\mathrm{OIII}] \lambda 5007$ line flux ratio, we are able to classify this source as a Seyfert $1.5 \mathrm{AGN}$ at redshift $0.0374 \pm 0.0003$. This is consistent with the redshift determination of Schneider et al. (2003) for this galaxy. This object was reported by Winter et al. (2008) as a Seyfert 1 galaxy.

\subsection{Swift J0911.2+4533}

The optical counterpart of this Swift source is the galaxy LEDA 2265450 , with a magnitude $B=16.43$ and a redshift of 0.0268 . Its radio counterpart is NVSS $091129+452804$ with a flux of $3.1 \pm 0.5 \mathrm{mJy}$.

The optical spectrum (Fig. 5, upper right panel), shows a very narrow emission $\mathrm{H}_{\alpha}+[\mathrm{NII}]$ complex, with the presence of [OIII] and [OII] forbidden emission lines. From these features we can classify this source as a Seyfert 2 AGN with a redshift of $0.0269 \pm 0.0003$. Our classification agrees with that reported in the BAT survey of Tueller et al. (2008).

\subsection{Swift J0917.2-6221}

The optical counterpart of this object is the galaxy LEDA 90443 , with redshift 0.0573 and magnitude $R=11.5$ reported in the USNO-A2.0 catalog. This source is associated with a ROSAT all-sky survey bright source (1RXS J091609.5-621934) and with the far-infrared object IRAS 09149-6206. Moreover, this Swift object is listed in the radio catalog MGPS-2 as MGPS-2 J091609-621928 with a flux at $843 \mathrm{MHz}$ of $61.4 \pm 2.1 \mathrm{mJy}$.

The spectrum (see Fig. 5, central left panel) shows very broad Balmer lines in emission, on a blue continuum, $\mathrm{H}_{\alpha}+[\mathrm{NII}]$ complex, as well as narrow [OIII] forbidden lines. Through the $\mathrm{H}_{\beta} /[\mathrm{O} \mathrm{III}] \lambda 5007$ line flux ratio, we can give a more accurate Seyfert 1.2 classification with respect to the Sy 1 given by Winter et al. (2008). The redshift calculated from the [O III] $\lambda 5007$ forbidden line is $0.057 \pm 0.001$, in agreement with that reported in Hyperleda archive. The source redshift implies a $843 \mathrm{MHz}$ radio luminosity of $4.7 \times 10^{39} \mathrm{erg} \mathrm{s}^{-1}$.

\subsection{Swift J0923.7+2255}

This object is associated with the galaxy LEDA 26614, with magnitude $B=15.16$ and redshift 0.0323 . It is listed in
Table 4. BLR gas velocities and central black hole masses for 6 Seyfert 1 AGNs listed in this paper.

\begin{tabular}{ccc}
\hline \hline Object & $\begin{array}{c}v_{\text {BLR }} \\
\left(\mathrm{km} \mathrm{s}^{-1}\right)\end{array}$ & $\begin{array}{c}M_{\mathrm{BH}} \\
\left(10^{7} M_{\odot}\right)\end{array}$ \\
\hline Swift J0059.4+3150 & 3500 & 4.5 \\
Swift J0342.0-2115 & 4150 & 8.3 \\
Swift J0743.0-2543 & 2510 & 2.6 \\
Swift J0904.3+5538 & 2910 & 3.3 \\
Swift J0917.2-6221 & 3460 & 7.0 \\
Swift J0923.7+2255 & 1820 & 6.4 \\
\hline
\end{tabular}

the ROSAT Bright Source Catalog (1RXS J092343.0+225437) and in the $X M M$ Serendipitous Source Catalog (2XMMi J092342.9+225433). It has an NVSS radio counterpart (NVSS $092343+225430$ ), with a flux density at $1.4 \mathrm{GHz}$ of $10.3 \pm$ $0.5 \mathrm{mJy}$. The spectrum (see Fig. 5, central right panel) shows the $\mathrm{H}_{\alpha}+[\mathrm{NII}]$ complex in emission, the Balmer lines, HeI permitted and some forbidden narrow lines in emission, Indeed, Swift J0923.7+2255 has a full width at half-maximum $(F W H M)$ of $\mathrm{H}_{\beta}$ line of $\sim 2000 \mathrm{~km} \mathrm{~s}^{-1}$, the presence of [FeI] emission lines and the $[\mathrm{OIII}]_{5007} / \mathrm{H}_{\beta}$ flux ratio $<3$. Following the approach of Osterbrock \& Pogge (1985) and Goodrich (1989) we are able to classify it as a Narrow-Line Seyfert $1 \mathrm{AGN}$, at $z=0.034 \pm 0.001$. Tueller et al. (2009) classified this source as a Seyfert 1.2 AGN.

\subsection{Swift J1049.4+2258}

The optical counterpart of this X-ray object is the galaxy LEDA 32398 (Mrk 417), with a $B$ magnitude of 16.21 and redshift 0.0326 . It is associated with a XMM 2nd serendipitous source (2XMMi J104930.9+225753).

The spectrum (Fig. 5, lower panel) shows a weak $\mathrm{H}_{\alpha}+[\mathrm{NII}]$ complex, narrow permitted $\mathrm{H}_{\beta}$ line and prominent [OIII] forbidden emission lines, with a weak continuum. Therefore, we classify this object as a Seyfert 2 AGN with redshift $0.033 \pm 0.001$, confirming what reported by Winter et al. (2008).

\subsection{Central black hole masses}

In this section we estimate the mass of the central black hole for 6 out of 7 type 1 AGNs. The method used here follows the prescription of Wu et al. (2004) and Kaspi et al. (2000). We used the $\mathrm{H}_{\beta}$ emission flux, corrected for the Galactic color excess (Schlegel et al. 1998), and the broad-line region (BLR) gas velocity.

Through Eq. (2) of Wu et al. (2004) we estimate the BLR size, then we used Eq. (5) of Kaspi et al. (2000) using the BLR size and the $v_{F W H M}$ to calculate the AGN black hole mass. The results are reported in Table 4 . We could not estimate the mass of the central black hole of Swift J0727.5-2406 because only the narrow component of the $\mathrm{H}_{\beta}$ line is detected for this source.

\subsection{Compton thickness estimation for type 2 AGNs}

Of the 17 extragalactic objects identified in this work, $9(\sim 53 \%)$ are type 2 Seyfert galaxies. In the Swift/BAT surveys as well as that of INTEGRAL/IBIS there are a number of highly absorbed Seyfert galaxies, among these there are a few Compton thick objects. For this reason in this section we try to investigate the nature of our type 2 objects by using a diagnostic tool developed by Malizia et al. (2007). 


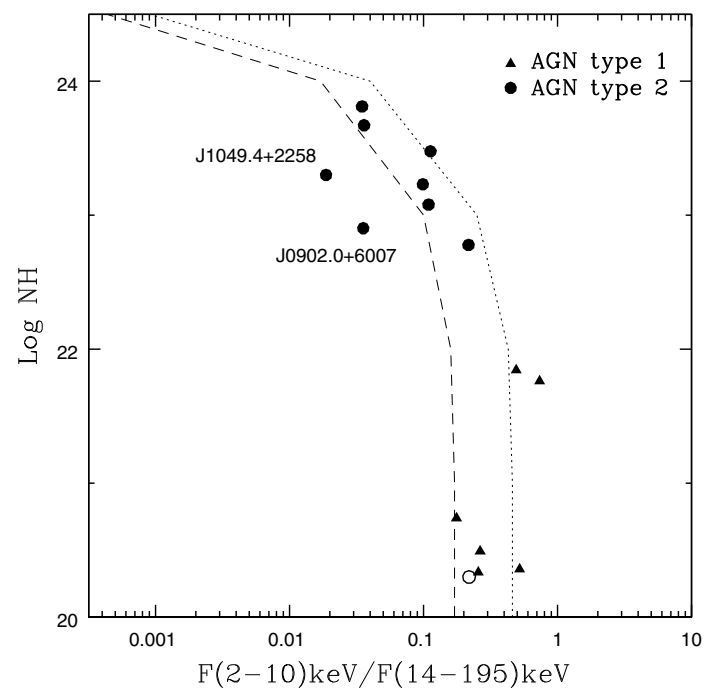

Fig. 7. $F_{2-10 \mathrm{keV}} / F_{14-195 \mathrm{keV}}$ flux ratio of our sample. Lines correspond to expected values for an absorbed power law with photon index 1.5 (dot) and 1.9 (dash). The open circle shows the position of Swift J0501.93239 , the Seyfert 2 without local absorption.

We have performed the X-ray data analysis of all our sample sources (see Table 3), in order to evaluate in a consistent way the absorption amount in excess to the Galactic one and the flux in the $2-10 \mathrm{keV}$ energy band. In a similar manner to Malizia et al. (2007), plotting the absorption against the softness ratio $F_{(2-10) \mathrm{keV}} / F_{(14-195) \mathrm{keV}}$ can help us to identify Compton thick candidates (see Fig. 7). A clear trend of decreasing softness ratio as the absorption increases is visible as expected if the $2-10 \mathrm{keV}$ flux is progressively depressed as the absorption becomes stronger. In Fig. 7 the value of $N_{\mathrm{H}}$ used is that of the intrinsic absorption, when measured (see Table 3), or the Galactic value in the cases either when no intrinsic absorption exists (mainly Seyfert 1 AGNs) or the statistics are too poor for it to be measured (Swift J0727.5-2406 and Swift J0811.5+0937). Lines represent the expected values for an absorbed power law with photon index of 1.5 (dotted line) and 1.9 (dashed line).

Most of our sources follow the expected trend, none of them turns out to have a low softness ratio with respect to the estimated column density; only two (Swift J0902.0+6007 and Swift J1049.4+2258) are slightly outside the expected trend, but probably in both cases the low quality statistics of the XRT data do not allow us to properly measure their column densities. It is important to note that, as expected the type 1 objects occupy the low part of the diagram (triangles in the figure), while the majority of our type 2 objects (circles) turn out to be highly absorbed $\left(>10^{23} \mathrm{~cm}^{-2}\right)$ AGNs; none of which appears to be a Compton thick object.

As reported in the Appendix a good quality XMM-Newton spectral measurement was available only for Swift J0739.6-3144, thus enabling its nature to be defined. For the rest of the sources the quality of the X-ray data is quite low and future deep X-ray observations, possibly together with high energy $(>10 \mathrm{keV})$ data, are needed to assess their nature. Finally, Swift J0501.9-3239 (open circle) is intriguing, since as reported in Sect. 3.6 we find this source to be a Seyfert 2, but from our XRT data analysis no absorption intrinsic to the source has been measured. Its softness ratio $(\sim 0.26)$ is not particularly low, therefore it is unlikely to be a Compton thick AGN. Also in this case higher X-ray data quality are needed to assess if it is a real unabsorbed Seyfert 2 galaxy.

\section{Conclusions}

In this work we have either given for the first time, or confirmed, or corrected, the optical spectroscopic identification of 17 Swift AGNs. This was achieved through a multisite observational campaign in Europe, Central and South America.

We found that our sample is composed of 16 AGNs (7 of type 1 and 9 of type 2) and 1 XBONG, with redshifts between 0.012 and 0.286 . For all our sources the X-ray data analysis has been performed in order to evaluate their main spectral parameters $\left(\Gamma, N_{\mathrm{H}}\right.$ and $2-10 \mathrm{keV}$ flux $)$. The measurements of the column densities and the soft X-ray fluxes, together with the hard X-ray ones provided by Swift-BAT, allowed us to use the diagnostic tool developed by Malizia et al. (2007) in order to pinpoint Compton thick candidates. Our Seyfert 2 objects turn out to be highly absorbed $\left(N_{\mathrm{H}} \sim 10^{23} \mathrm{~cm}^{-2}\right)$, but none of them are Compton thick.

Moreover, for six type 1 AGNs we have estimated the BLR size, velocity and the central black hole mass.

All of the results shown in this work stress the importance of the optical spectroscopic followup, not only for the classification of unidentified sources and for the study of the statistical properties of the various source classes, but also for the search of Compton thick AGNs, that are thought to provide an important contribution to the overall cosmic energy budget at hard X-rays, but for which the cosmological evolution and space density are not well known yet.

Acknowledgements. We thank Silvia Galleti for Service Mode observations at the Loiano telescope; Francesca Ghinassi for service mode observations at the TNG; Hripsime Navasardyan for service mode observations at the Asiago Telescope; Antonio De Blasi and Ivan Bruni for night assistance at the Loiano telescope; Edgardo Cosgrove, Manuel Hernández and José Velásquez for day and night assistance at the CTIO telescope. We also thank the anonymous referee for useful comments which helped us to improve the quality of this paper. This research has made use of the ASI Science Data Center Multimission Archive, of the NASA Astrophysics Data System Abstract Service, the NASA/IPAC Extragalactic Database (NED), of the NASA/IPAC Infrared Science Archive, which are operated by the Jet Propulsion Laboratory, California Institute of Technology, under contract with the National Aeronautics and Space Administration and of data obtained from the High Energy Astrophysics Science Archive Research Center (HEASARC), provided by NASA's GSFC. This publication made use of data products from the Two Micron All Sky Survey (2MASS), which is a joint project of the University of Massachusetts and the Infrared Processing and Analysis Center/California Institute of Technology, funded by the National Aeronautics and Space Administration and the National Science Foundation. This research has also made use of data extracted from the $6 \mathrm{dF}$ Galaxy Survey and the Sloan Digitized Sky Survey archives; the SIMBAD database operated at CDS, Strasbourg, France, and of the HyperLeda catalogue operated at the Observatoire de Lyon, France. The authors acknowledge the ASI and INAF financial support via grants No. I/023/05/0 and I/008/07; P.P. is supported by the ASI-INTEGRAL grant No. I/008/07. L.M. is supported by the University of Padua through grant No. CPDR061795/06. G.G. is supported by FONDECYT 1085267. V.C. is supported by the CONACYT research grant 54480-F (México). D.M. is supported by the Basal CATA PFB 06/09, and FONDAP Center for Astrophysics grant No. 15010003.

\section{References}

Adelman-McCarthy, J. K., Agüeros, M. A., Allam, S. S., et al. 2007, ApJS, 172, 634

Ajello, M., Rau, A., Greiner, J., et al. 2008, ApJ, 673, 96

Barthelmy, S. D. 2004, Proc. SPIE, 5165, 175

Bassani, L., Malizia, A., Stephen, J. B., et al. 2006 [arXiv: astro-ph/0610455v1]

Beckmann, V., Soldi, S., Shrader, C. R., et al. 2006, ApJ, 652, 126 Bianchi, S., Corral, A., Panessa, F., et al. 2008, MNRAS, 385, 195 Bikmaev, I. F., Sunyaev, R. A., \& Revnivtsev, M. G. 2006, Astron. Lett., 32, 221 Bird, A. J., Malizia, A., Bazzano, A., et al. 2007, ApJS, 170, 175 Burrows, D. N., Hill, J. E., Nousek, J. A., et al. 2004, Proc. SPIE, 5165, 201 Burrows, D. N., Hill, J. E., Nousek, J. A., et al. 2005, Space Sci. Rev., 120, 165 Cardelli, J. A., Clayton, G. C., \& Mathis, J. S. 1989, ApJ, 345, 245 
Comastri, A. 2004, Supermassive Black Holes in the Distant Universe, ed. A. L. Barger (Dordrecht, The Netherlands: Kluwer Academic Publishers), Astrophys. Space Sci. Lib., 308, 245

Comastri, A., Mignoli, M., Ciliegi, P., et al. 2002, ApJ, 571, 771

Condon, J. J., Cotton, W. D., Greisen, E. W., et al. 1998, AJ, 115, 1693

Cusumano, G., La Paola, V., Segreto, A., et al. 2009, A\&A, accepted [arXiv: 0906.4788]

Doyle, M. T., Drinkwater, M. J., Rohde, D. J., et al. 2005, MNRAS, 361, 34

Dressler, A., \& Shectman, S. 1987, AJ, 94, 899

Elitzur, M. 2008, New Astron. Rev., 52, 274

Gehrels, N., Chincarini, G., Giommi, P., et al. 2004, ApJ, 611, 1005

Ghisellini, G., Haardt, F., Matt, G. 1994, MNRAS, 267, 743

Giommi, P., Perri, M., \& Fiore, F. 2001, A\&A, 362, 799

Goodrich, R. W. 1989, ApJ, 342, 224

Guainazzi, M., \& Bianchi, S. 2007, MNRAS, 374, 1290

Guainazzi, M., Matt, G., \& Perola, G. C. 2005, A\&A, 444, 119

IRAS 1988, The Point Source Catalog, version 2.0, NASA RP-1190

Hill, J. E., Burrows, D. N., Nousek, J. A., et al. 2004, Proc. SPIE, 5165, 217

Ho, L. C., Filippenko, A. V., \& Sargent, W. L. W. 1993, ApJ, 417, 63

Ho, L. C., Filippenko, A. V., \& Sargent, W. L. W. 1997, ApJS, 112, 315

Horne, K. 1986, PASP, 98, 609

Ishisaki, Y., Ueda, Y.,Yamashita, A., et al. 2001, ASPC, 251, 458

Iwasawa, K., Wilson, A. S., Fabian, A. C., et al. 2003, MNRAS, 345, 369

Jones, D. H., Saunders, W., Colless, M., et al. 2004, MNRAS, 355, 747

Jones, D. H., Saunders, W., Read, M., et al. 2005, PASA, 22, 277

Kaspi, S., Smith, P. S., Netzer, H., et al. 2000, ApJ, 533, 631

Kauffmann, G., Heckman, T. M., Tremonti, C., et al. 2003, MNRAS, 346, 1055

Krivonos, R., Revnivtsev, M., Lutovinov, A., et al. 2007, A\&A, 475, 775

Laurent-Muehleisen, S. A., Kollgaard, R. I., Ciardullo, R., et al. 1998, ApJS, 118,127

Malizia, A., Landi, R., Bassani, L., et al. 2007, ApJ, 668, 81

Malizia, A., Bassani, L., Panessa, F., et al. 2009, MNRAS, 394, 121

Markwardt, C. B., Tueller, J., Skinner, G. K., et al. 2005, ApJ, 633, L77

Masetti, N., Palazzi, E., Bassani, L., et al. 2004, A\&A, 426, L41

Masetti, N., Bassani, L., Bazzano, A., et al. 2006a, A\&A, 455, 11

Masetti, N., Morelli, L., Palazzi, E., et al. 2006b, A\&A, 459, 21

Masetti, N., Mason, E., Morelli, L., et al. 2008, A\&A, 482, 113

Masetti, N., Parisi, P., Palazzi, E., et al. 2009, A\&A, 495, 121
Matt, G., Fabian, A. C., Guainazzi, M., et al. 2000, MNRAS, 318, 173 Mauch, T., Murphy, T., Buttery, H. J., et al. 2003, MNRAS, 342, 1117 Monet, D. G., Levine, S. E., Canzian, B., et al. 2003, AJ, 125, 984

Moretti, A., Campana, S., Tagliaferri, G., et al. 2004, SPIE Proc., 5165, 232

Murphy, T., Mauch, T., Green, A., et al. 2007, MNRAS, 382, 382

Osterbrock, D. E. 1989, Astrophysics of Gaseous Nebulae and Active Galactic Nuclei (Mill Valley: Univ. Science Books)

Osterbrock, D. E., \& Pogge, R. W. 1985, ApJ, 297, 166

Paltani, S., Walter, R., McHardy, I. M., et al. 2008, A\&A, 485, 707

Press, W. H., Teukolsky, S. A., Vetterling W. T., et al. 1992, Numerical Recipes (Cambridge: Cambridge Univ. Press)

Prugniel, P. 2005, The Hyperleda Catalogue http: //leda.univ-lyon1.fr/ Revnivtsev, M., Sazonov, S. Y., Jahoda, K., et al. 2004, A\&A, 418, 927

Revnivtsev, M., Sazonov, S. Y., Churazov, E., et al. 2006, A\&A, 448, L49

Romano, P., Campana, S., Chincarini, G., et al. 2006, A\&A, 456, 917

Roming, P. W. A., Hunsberger, S. D., Mason, K. O., et al. 2004, Proc. SPIE, 5165,262

Sazonov, S., Revnivtsev, M., Krivonos, R., et al. 2007, A\&A, 462, 57

Saxton, R. D., Read, A. M., Esquej, P., et al. 2008, A\&A, 480, 611

Schlegel, D. J., Finkbeiner, D. P., \& Davis, M. 1998, ApJ, 500, 525

Schneider, D. P., Fan, X., Hall, P. B., et al. 2003, AJ, 126, 2579

Skrutskie, M. F., Cutri, R. M., Stiening, R., et al. 2006, AJ, 131, 1163

Tueller, J., Mushotzky, R. F., Barthelmy, S., et al. 2008, ApJ, 681, 113

Tueller, J., Baumgartner, W. H., Markwardt, C. B., et al. 2009, ApJS, in press [arXiv: 0903.3037]

Ubertini, P., Lebrun, F., Di Cocco, G., et al. 2003, A\&A, 411, L131

Ueda, Y., Takahashi, T., \& Ishisaki, Y. 1999, ApJ, 524, 11

Ueda, Y., Eguchi, S., Terashima, Y., et al. 2007, ApJ, 665, 209

Veilleux, S., \& Osterbrock, D. E. 1987, ApJS, 63, 295

Voges, W., Aschenbach, B., Boller, T., et al. 1999, A\&A, 349, 389

Watson, M. G., Schröder, A. C., Fyfe, D., et al. 2009, A\&A, 493, 339

Winkler, H. 1992, MNRAS, 257, 677

Winkler, C., Courvoisier, T. J.-L., Di Cocco, G., et al. 2003, A\&A, 411, L1

Winter, L. M., Mushotzky, R. F., Tueller, J., et al. 2008, ApJ, 674, 686

Wright, E. L. 2006, PASP, 118, 1711

Wu, X.-B., Wang, R., Kong, M. Z., Liu, F. K., \& Han, J. L. 2004, A\&A, 424, 793 